\title{
Measurement of Thresholds Using Auditory Steady- State Response and Cochlear Microphonics in Children with Auditory Neuropathy
}

DOI: $10.3766 /$ jaaa.17013

\author{
Ping $\mathrm{Lu}^{*}+$ \\ Yue Huang* \\ Wen-Xia Chen* \\ Wen Jiang $\dagger$ \\ Ni-Yi Hua* \\ Yan Wang* \\ Bin Wang* \\ Zheng-Min $\mathrm{Xu}^{*}$
}

\begin{abstract}
Background: The detection of precise hearing thresholds in infants and children with auditory neuropathy (AN) is challenging with current objective methods, especially in those younger than six months of age.

Purpose: The aim of this study was to compare the thresholds using auditory steady-state response (ASSR) and cochlear microphonics (CM) in children with $\mathrm{AN}$ and children with normal hearing.

Research Design: The thresholds of CM, ASSR, and visual reinforcement audiometry (VRA) tests were recorded; the ASSR and VRA frequencies used were 250, 500, 1000, 2000, and $4000 \mathrm{~Hz}$.

Study Sample: The participants in this study were 15 children with AN (27 ears) (1-7.6 years, median age 4.1 years) and ten children with normal hearing (20 ears) (1-8 years, median age four years).

Data Collection and Analysis: The thresholds of the three methods were compared, and histograms were used to represent frequency distributions of threshold differences obtained from the three methods.

Results: In children with normal hearing, the average CM thresholds ( $84.5 \mathrm{~dB}$ ) were significantly higher than the VRA thresholds (10.0-10.8 dB); in children with AN, both CM and VRA responses were seen at high signal levels (88.9 dB and 70.6-103.4 dB, respectively). In normal children, the difference between mean VRA and ASSR thresholds ranged from 17.5 to $30.3 \mathrm{~dB}$, which was significantly smaller than the difference seen between the mean CM and VRA thresholds (71.5-72.3 dB). The correlation between VRA and ASSR in children with normal hearing ranged from 0.38 to 0.48 , whereas no such correlation was seen in children with $\mathrm{AN}$ at any frequency $(0.03-0.19)$.
\end{abstract}

Conclusions: Our results indicated that ASSR and CM were poor predictors of the conventional behavioral threshold in children with AN.

Key Words: auditory neuropathy, auditory steady-state response, cochlear microphonics

Abbreviations: $A B R=$ auditory brainstem response test; $A N=$ auditory neuropathy; $A S S R=$ auditory steady-state response; $\mathrm{CM}=$ cochlear microphonics; $\mathrm{OAE}=$ otoacoustic emissions; $\mathrm{OHC}=$ outer hair cell; VRA = visual reinforcement audiometry

*Department of Otolaryngology-Head and Neck Surgery, Children’s Hospital of Fudan University, Shanghai, China; †Xuzhou Medical University, Xuzhou, China; $¥$ These authors contributed equally to this work.

Corresponding author: Zheng-Min Xu, Department of Otolaryngology-Head and Neck Surgery, Children's Hospital of Fudan University, Shanghai 201102, China; Email: 13916320945@163.com 


\section{INTRODUCTION}

A uditory neuropathy (AN), AN/auditory dyssynchrony (AD) (Berlin et al, 2001), and more recently defined AN spectrum disorder (Roush et al, 2011) are variable terms used to describe an abnormal hearing condition seen in infants and adults, and the condition was first reported by Starr et al (1996). It is characterized by normal outer hair cell (OHC) function and abnormal neural function at the level of the eighth nerve, as exemplified by the presence of otoacoustic emissions (OAEs) and/or cochlear microphonics (CM), and abnormal auditory brainstem response test (ABR), respectively (Berlin et al, 1998). The occurrence of $\mathrm{AN}$ is more frequent than initially believed, and represents as much as $8 \%$ of the newly diagnosed pediatric deafness cases in the neonatal intensive care unit (Rea and Gibson, 2003; Berg et al, 2005).

$\mathrm{CM}$ is a small, alternating current potential produced by OHCs and represents the early components of the ABR (Withnell, 2001). Although discovered in the 1930s, CM was not used widely in clinics because of difficulties in detection. With the advancement of medical technologies, $\mathrm{CM}$ now plays an important role in the diagnosis of AN, and some recent studies (Rance et al, 1999; Starr et al, 2001; Buchman et al, 2006; Santarelli et al, 2006; Riazi and Ferraro, 2008) have revealed more $\mathrm{CM}$ characteristics in $\mathrm{AN}$ that have helped us understand the specific relevance of $\mathrm{CM}$.

In recent years, the auditory steady-state evoked response (ASSR) has emerged as an alternative electrophysiological technique for measuring objective thresholds in young children. Some studies have documented high variability in the relationship between ASSR and behavioral thresholds for normal-hearing and hearing-impaired infants, and the correlation is usually positive in cases of normal hearing and some sensorineural hearing loss (Yang et al, 2008; Ribeiro et al, 2010), but poor in some post-cochlear hearing loss cases (Rance et al, 1999; Rance and Briggs, 2002).

Several studies have suggested that intervention before the age of six months can increase the possibility of normal speech and language development (Pimperton and Kennedy, 2012), but the precise detection of hearing thresholds with current objective methods is still a challenge in children with AN younger than six months. Therefore, it is not easy to confirm whether appropriate intervention was provided for those children. In this study, the thresholds of $\mathrm{CM}$ and ASSR in children with AN were observed.

\section{PARTICIPANTS AND METHODS}

\section{Participants}

Fifteen children (27 ears) aged 1-7.6 years (median age 4.1 years) who were brought to the Otolaryngology
Head and Neck Surgery of Children's Hospital of Fudan University between January 2010 and July 2016 were observed; 11 children were girls and ten were boys. All the children were diagnosed with AN according to the following criteria (Berlin et al, 1998): (a) normal OHC function (presence of OAEs and/or CM) and (b) abnormal neural function at the level of the eighth nerve demonstrated by absent or highly abnormal ABRs. Ten children with normal hearing (20 ears) aged $1-8.0$ years (median age 4.0 years) were enrolled as control group, and all of them passed the OAE test and had a clickABR threshold of $\leq 30 \mathrm{~dB}$ nHL. For each participant, the CM, visual reinforcement audiometry (VRA), and ASSR mean threshold tests were carried out by an audiologist. CM thresholds were present in all AN cases, but not the VRA responses and ASSRs: ASSR was seen in $19,26,27,27$, and 25 ears at $250,500,1000,2000$, and $4000 \mathrm{~Hz}$, respectively, and VRA was present in 25,27 , 27,25 , and 19 ears at 250,500,1000,2000, and $4000 \mathrm{~Hz}$, respectively. The CM, VRA, and ASSR were present in all children with normal hearing. This study was approved by the Ethics Committee of the Children's Hospital of Fudan University and conducted in accordance with the ICH guidelines for Good Clinical Practice and the Declaration of Helsinki. The guardians of the children signed informed consent forms.

\section{Recording Methods of CM}

CM was performed in a soundproof room. All participants were sedated with $10 \%$ chloral hydrate at $0.5 \mathrm{ml} / \mathrm{kg}$ orally. CM was measured with a GSI Audera Brainstem Analyzer using a Model TIP-50 earphone. Silver disc electrodes were placed on the mastoid (active), nasion (ground), and forehead (reference), and a maximum stimulus level of $109.6 \mathrm{~dB}$ nHL was used to elicit the waveforms. The bandpass filter settings were 30 and $5000 \mathrm{~Hz}$ with a 10 -msec window, and average response was obtained twice by recording the response to two series of 2,006 clicks. The electrode impedance measured at any site was $<10 \mathrm{k} \Omega$, and the inter-electrode impedance was $<10 \mathrm{k} \Omega$. CM response appeared between the presence of a regular ABR waveform and the click stimulus, and inverted with stimulus polarity reversal, whereas neural responses to clicks typically did not completely invert (Figure 1A). Once it was assured that the measurements were reproducible, one run was performed with the tube clamped. The test was stopped when the software obtained a stable CM representation, after which the CM threshold was recorded (Hood, 2015).

\section{Recording Methods of ASSR}

The ASSR was recorded with a GSI Audera Brainstem Analyzer. Silver disc electrodes were placed on 

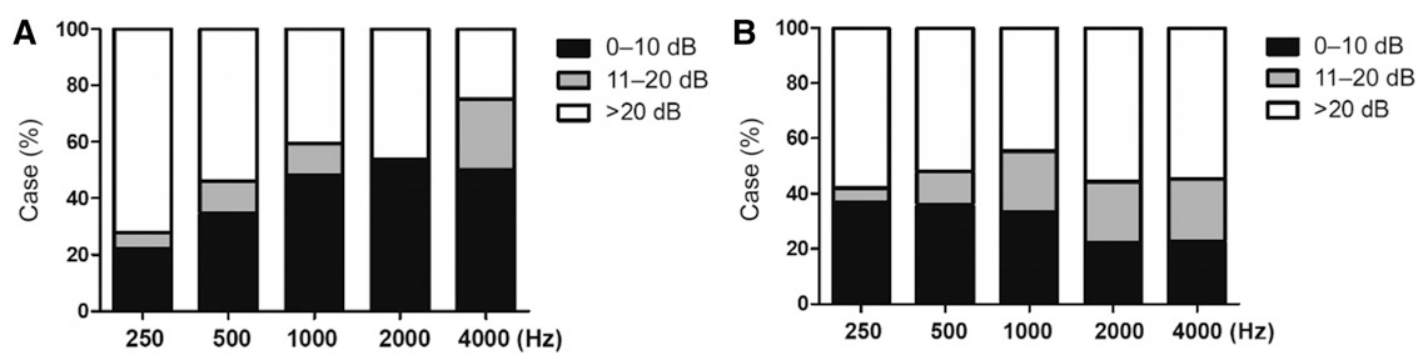

Figure 1. Histogram showing the percentage of cases vs. measurement difference between tests in three ranges: 0-10, 11-20, and $>20 \mathrm{~dB}$ HL for each tested frequency. (A) VRA/ASSR; (B) VRA/CM.

the participants' scalps in the following positions: nasion (ground electrode), forehead (active electrode), and mastoid (reference electrode). Dichotic stimulation and carrier frequencies of $250,500,1000,2000$, and $4000 \mathrm{~Hz}$ were used, and the modulation rates were $67,74,81,88$, and $95 \mathrm{~Hz}$. Both amplitude-modulated and frequency-modulated modes were used at depths of $100 \%$ and $10 \%$, respectively. The stimuli were applied through inserted earphones (Model TIP-50 earphone).

\section{Recording Methods of VRA}

VRA measurements were established for each ear in a soundproof room. The child sat in a chair, and the examiner assessed hearing using earphones in each ear separately. After emitting a sound at a specific frequency, the child's eye-shift or head-turn response toward the sound source was rewarded by activation of a lighted mechanical toy. The behavioral thresholds were obtained using conditioned audiometric techniques (visual response audiometry) with earphones, and $10 \mathrm{~dB}$ HL down- and $5 \mathrm{~dB}$ HL up-threshold were used at each procedure. Our clinical audiometer (Dianostic Audiometer AS229e; Denmark) was used to generate warble tones at the frequency range of 250, 500, 1000, 2000, and $4000 \mathrm{~Hz}$.

\section{Data Analysis}

The results were expressed as means \pm standard deviation, and histograms were used to represent frequency distributions of threshold differences obtained from three methods.

\section{RESULTS}

\section{Comparing VRA and CM Measurements in Children with Normal Hearing and Children with AN}

The means and standard deviations of the auditory thresholds measured by CM, VRA, and ASSR at each frequency are summarized in Table 1 . In children with normal hearing, CM responses were seen at higher signal levels (mean threshold is $84.5 \mathrm{~dB}$ ) compared with the VRA responses (mean threshold is 10.0-10.8 dB at each frequency), with a difference of $70 \mathrm{~dB}$ between the two measurements. In children with AN with severe-to-profound hearing loss, the mean threshold of $\mathrm{CM}$ responses at high signal levels was $88.9 \mathrm{~dB}$, and the mean thresholds of VRA were $70.6,73.7,85.9$, 92.2 , and $103.4 \mathrm{~dB}$ at $250,500,1000,2000$, and 4000 $\mathrm{Hz}$, respectively, bringing the difference between the measurements to only $15.9-29.4 \mathrm{~dB}$. A significant difference was observed in CM/VRA threshold difference between children with normal hearing and children with AN $(p<0.01)$.

Based on the CM/VRA threshold differences, the readings were classified into three groups: $0-10 \mathrm{~dB}$, $11-20 \mathrm{~dB}$, and $>20 \mathrm{~dB}$. As shown in Figure 1A, $27.8 \%, 46.2 \%, 59.3 \%, 53.8 \%$, and $75 \%$ of the test participants had CM/VRA threshold difference $<20 \mathrm{~dB}$ at $250,500,1000,2000$, and $4000 \mathrm{~Hz}$, respectively.

\section{Comparison of VRA and ASSR Measurements in Children with Normal Hearing and Children with AN}

In children with normal hearing, ASSR thresholds were slightly higher than those obtained from VRA at each frequency. The mean threshold difference between VRA and ASSR ranged from 17.5 to $30.3 \mathrm{~dB}$, which was significantly smaller than the mean threshold difference between CM and VRA (71.5-72.3 dB) (Table 1). As shown in Figure 1B, 36.8\%, 36.0\%, 33.3\%, $22.2 \%$, and $22.7 \%$ of the test participants had ASSR/VRA threshold difference $<10 \mathrm{~dB}$, and $5.3 \%, 12.0 \%, 22.2 \%, 22.2 \%$, and $22.7 \%$ of the participants had ASSR/VRA threshold difference between 11 and $20 \mathrm{~dB}$ at 250, 500, 1000, 2000, and $4000 \mathrm{~Hz}$, respectively.

Although no significant difference was observed in ASSR/VRA threshold difference between children with normal-hearing and children with $\mathrm{AN}(p>0.05)$, but the correlation between VRA and ASSR in children with 
CM and ASSR in Infants and Children with AN/Lu et al

Table 1. Mean Thresholds of CM, ASSR, and VRA in Children with Normal-Hearing and Children with AN According to Carrier Frequency

\begin{tabular}{|c|c|c|c|c|c|c|}
\hline & VRA & ASSR & $\mathrm{CM}$ & Difference $1^{*}$ & Difference $2^{\star}$ & Correlation $\dagger$ \\
\hline \multicolumn{7}{|c|}{ Children with normal hearing } \\
\hline $250 \mathrm{~Hz}$ & $10.0 \pm 4.3$ & $40.3 \pm 13.5$ & $84.5 \pm 10.7$ & $30.3 \pm 12.1$ & $72.3 \pm 9.0 \ddagger$ & 0.48 \\
\hline $500 \mathrm{~Hz}$ & $10.5 \pm 5.6$ & $38.0 \pm 10.9$ & & $27.5 \pm 9.8$ & $71.8 \pm 9.2 \ddagger$ & 0.45 \\
\hline $1000 \mathrm{~Hz}$ & $10.0 \pm 3.2$ & $28.3 \pm 8.6$ & & $18.3 \pm 7.7$ & $72.3 \pm 8.8 \ddagger$ & 0.47 \\
\hline $2000 \mathrm{~Hz}$ & $10.8 \pm 6.1$ & $28.8 \pm 11.0$ & & $18.0 \pm 10.1$ & $71.5 \pm 9.9 \ddagger$ & 0.42 \\
\hline $4000 \mathrm{~Hz}$ & $10.8 \pm 3.7$ & $28.3 \pm 9.4$ & & $17.5 \pm 8.7$ & $71.5 \pm 9.3 \ddagger$ & 0.38 \\
\hline \multicolumn{7}{|c|}{ Children with AN } \\
\hline $250 \mathrm{~Hz}$ & $70.6 \pm 17.2$ & $83.9 \pm 21.6$ & $88.9 \pm 8.2$ & $29.4 \pm 22.2$ & $22.1 \pm 21.3 \S$ & 0.03 \\
\hline $500 \mathrm{~Hz}$ & $73.7 \pm 22.3$ & $87.9 \pm 23.9$ & & $25.6 \pm 20.1$ & $22.0 \pm 15.5 \S$ & 0.16 \\
\hline $1000 \mathrm{~Hz}$ & $85.9 \pm 25.8$ & $93.0 \pm 18.9$ & & $20.4 \pm 21.4$ & $23.7 \pm 19.8 \S$ & 0.19 \\
\hline $2000 \mathrm{~Hz}$ & $92.2 \pm 18.9$ & $93.5 \pm 19.6$ & & $17.0 \pm 17.1$ & $18.3 \pm 17.7 \S$ & 0.16 \\
\hline $4000 \mathrm{~Hz}$ & $103.4 \pm 17.2$ & $102.2 \pm 16.7$ & & $15.9 \pm 16.2$ & $22.3 \pm 13.7 \S$ & 0.15 \\
\hline
\end{tabular}

*Difference 1 means the differences between VRA and ASSR thresholds; difference 2 means the differences between CM and VRA thresholds. †Correlation means the correlation of VRA and ASSR thresholds.

\$Difference 1 versus difference 2 in children with normal hearing, $p<0.01$.

§Difference 2 between children with normal hearing and children with $A N, p<0.01$.

normal hearing ranged from 0.38 to 0.48 , whereas no significant correlation was between VRA and ASSR in children with $\mathrm{AN}(0.03-0.19)$ at any frequency, which indicated that ASSR was a poor predictor of the conventional behavioral threshold in these participants.

\section{DISCUSSION}

$\mathrm{T}$ he hallmark of AN is a negligible or very abnormal $\mathrm{ABR}$ reading along with a normal OAE reading, the latter indicating normal $\mathrm{OHC}$ function (Hood, 2015). However, not every patient with AN can pass the OAE detection, and the passing rate in our study was $42.4 \%$. Although $\mathrm{CM}$ is usually present despite the loss of OAEs, it is more stable in some conditions (Hood, 2015). CM is obtained by recording transtympanic or extra-tympanic electro-cochleography, but the current simpler detection method uses surface recording by means of skin electrodes. The CM is easily recorded from standard click-evoked ABR recording protocols when insert earphones are used, but highlevel click stimuli (80-90 dB nHL) are necessary (Starr et al, 2001) because of the distance between the active electrode and the source of CM generation. Consistent with this, the CM thresholds in our study were $84.5 \pm$ 10.7 and $88.9 \pm 8.2$ in normal infants and children and those with $\mathrm{AN}$, respectively.

Pure tone audiometry (PTA) is the key hearing test used to identify hearing threshold levels of an individual, and helps determine the degree, type, and configuration of a hearing loss, but it cannot be used in infants and unwilling patients. Poch-Broto et al (2000) found that audiometric profiles obtained from CM are highly correlated with those obtained with PTA. More than $81 \%$ of patients exhibited differences $<10 \mathrm{~dB}$ (HL) between tests at all tested frequencies
(91.67\% at $250 \mathrm{~Hz}$ ). Their study included 60 adult patients with conductive hypoacusis, neurosensoryrelated hypoacusis, or normoacusis PTA patterns, and measured CM by a cochlear microphonics potential recording method described previously, which is different from the method we used in our study. Although the threshold differences of PTA and CM ranged from 18.3 to 23.7 , about $50 \%$ of individuals with $\mathrm{AN}$ in our study showed threshold differences $<20 \mathrm{~dB}$ at each frequency. This may be because of the fact that all children with AN included in our study had severe-to-profound hearing loss and had cochlear implants, and that CM responses occurred at high signal levels in both children with AN and those with normal hearing. Therefore, we believe that CM threshold is not a suitable method to estimate auditory threshold in children with AN.

Studies on ASSR show that in persons with normal hearing, ASSR has good predictive value for behavioral hearing thresholds, whereas the pure-tone sensitivity and ASSR threshold are not correlated in individuals afflicted with AN (Rance, 2005; Attias et al, 2006; Jafari et al, 2009; McCreery and Simmons, 2011). Other studies have reported ASSR thresholds at $80 \mathrm{~dB}$ and greater, regardless of pure-tone findings, in AN cases (Attias et al, 2006; Rance et al, 1998), which negates the use of ASSR threshold in judging the "severity" of AN hearing loss. In our study, the difference in VRA and ASSR mean thresholds ranged from 17.5 to $30.3 \mathrm{~dB}$ in children with normal hearing, consistent with Lins et al (1996). The correlation between VRA and ASSR in children with normal hearing was between 0.38 and 0.47 at different frequencies, slightly lower than previous reports on normal adults (0.72-0.98). Although the difference between VRA and ASSR mean thresholds in the children with $\mathrm{AN}$ ranged from 18.3 to $13.7 \mathrm{~dB}$, the correlation between VAR and ASSR was weak (0.03-0.19) at most 
of the frequencies usually tested in children with AN. This is consistent with the findings of Jafari et al (2009) on 16 adults (32 ears) with AN.

In conclusion, the findings of the present study indicate that ASSR or CM is unsuitable for estimating the auditory thresholds of individuals suspected of having AN.

\section{REFERENCES}

Attias J, Buller N, Rubel Y, Raveh E. (2006) Multiple auditory steady-state responses in children and adults with normal hearing, sensorineural hearing loss, or auditory neuropathy. Ann Otol Rhinol Laryngol 115:268-276.

Berg AL, Spitzer JB, Towers HM, Bartosiewicz C, Diamond BE. (2005) Newborn hearing screening in the NICU: profile of failed auditory brainstem response/passed otoacoustic emission. Pediatrics 116:933-938.

Berlin C, Hood L, Rose K. (2001) On renaming auditory neuropathy as auditory dys-synchrony. Audiology Today 13:15-17.

Berlin CI, Bordelon J, St John P, Wilensky D, Hurley A, Kluka E, Hood LJ. (1998) Reversing click polarity may uncover auditory neuropathy in infants. Ear Hear 19:37-47.

Buchman CA, Roush PA, Teagle HF, Brown CJ, Zdanski CJ, Grose JH. (2006) Auditory neuropathy characteristics in children with cochlear nerve deficiency. Ear Hear 27:399-408.

Hood LJ. (2015) Auditory neuropathy/dys-synchrony disorder: diagnosis and management. Otolaryngol Clin North Am 48:1027-1040.

Jafari Z, Malayeri S, Ashayeri H, Farahani MA. (2009) Adults with auditory neuropathy: comparison of auditory steady-state response and pure-tone audiometry. J Am Acad Audiol 20:621-628.

Lins OG, Picton TW, Boucher BL, Durieux-Smith A, Champagne SC, Moran LM, Perez-Abalo MC, Martin V, Savio G. (1996) Frequencyspecific audiometry using steady-state responses. Ear Hear 17: 81-96.

McCreery R, Simmons J. (2011) Auditory steady state response in auditory neuropathy. J Laryngol Otol 125:324-325; author reply 325

Pimperton H, Kennedy CR. (2012) The impact of early identification of permanent childhood hearing impairment on speech and language outcomes. Arch Dis Child 97:648-653.

Poch-Broto J, Carricondo F, Bhathal B, Iglesias MC, LopezMoya J, Rodriguez F, Sanjuan J, Gil-Loyzaga P. (2009) Cochlear microphonic audiometry: a new hearing test for objective diagnosis of deafness. Acta Otolaryngol 129:749-754.

Rance G. (2005) Auditory neuropathy/dys-synchrony and its perceptual consequences. Trends Amplif 9:1-43.

Rance G, Beer DE, Cone-Wesson B, Shepherd RK, Dowell RC, King AM, Rickards FW, Clark GM. (1999) Clinical findings for a group of infants and young children with auditory neuropathy. Ear Hear 20:238-252.

Rance G, Briggs RJ. (2002) Assessment of hearing in infants with moderate to profound impairment: the Melbourne experience with auditory steady-state evoked potential testing. Ann Otol Rhinol Laryngol Suppl 189:22-28.

Rance G, Dowell RC, Rickards FW, Beer DE, Clark GM. (1998) Steady-state evoked potential and behavioral hearing thresholds in a group of children with absent click-evoked auditory brain stem response. Ear Hear 19:48-61.

Rea PA, Gibson WP. (2003) Evidence for surviving outer hair cell function in congenitally deaf ears. Laryngoscope 113:2030-2034.

Riazi M, Ferraro JA. (2008) Observations on mastoid versus ear canal recorded cochlear microphonic in newborns and adults. $J$ Am Acad Audiol 19:46-55.

Ribeiro FM, Carvallo RM, Marcoux AM. (2010) Auditory steadystate evoked responses for preterm and term neonates. Audiol Neurootol 15:97-110.

Roush P, Frymark T, Venediktov R, Wang B. (2011) Audiologic management of auditory neuropathy spectrum disorder in children: a systematic review of the literature. Am J Audiol 20: $159-170$

Santarelli R, Scimemi P, Dal Monte E, Arslan E. (2006) Cochlear microphonic potential recorded by transtympanic electrocochleography in normally-hearing and hearing-impaired ears. Acta Otorhinolaryngol Ital 26:78-95.

Starr A, Picton TW, Sininger Y, Hood LJ, Berlin CI. (1996) Auditory neuropathy. Brain 119:741-753.

Starr A, Sininger Y, Nguyen T, Michalewski HJ, Oba S, Abdala C. (2001) Cochlear receptor (microphonic and summating potentials, otoacoustic emissions) and auditory pathway (auditory brain stem potentials) activity in auditory neuropathy. Ear Hear 22:91-99.

Withnell RH. (2001) Brief report: the cochlear microphonic as an indication of outer hair cell function. Ear Hear 22:75-77.

Yang CH, Chen HC, Hwang CF. (2008) The prediction of hearing thresholds with auditory steady-state responses for cochlear implanted children. Int J Pediatr Otorhinolaryngol 72:609-617. 